\title{
Health Education in the Healthy China Initiative 2019-2030
}

\author{
Zhuojun Jiang'; Wen Jiang ${ }^{1, *}$
}

\section{Summary}

As the first of 15 Healthy China initiatives, the Health Education Initiative has the crucially important goal of improving citizens' health literacy. There are two key activities in the initiative for improving health literacy. The first is to establish a mechanism for dissemination of health sciences knowledge through development of national and provincial databases of health sciences expertise and a national resource of accessible health knowledge. The second is to establish a health education and health promotion performance evaluation mechanism for medical institutions and medical personnel. In this paper, we analyzed the content and strategies of these two health educational activities.

\section{BACKGROUND}

In 2016, China put forward "Healthy China 2030" as a national strategy, stressing the importance that China places people's health as a developmental priority (1) and the importance of China's active participation in global health governance and fulfillment of its commitment to the United Nation's 2030 Agenda for Sustainable Development (2). Three years later, China promulgated the Healthy China Initiative 2019-2030 as the implementation plan for Healthy China 2030 (3).

The Healthy China Initiative 2019-2030 will conduct 15 major initiatives, including health education promotion, mental health promotion, and tobacco control, each with its own progress indicators. These initiatives highlight the importance of preventing and controlling diseases at their source, targeting major problems and diseases affecting people's health, and highlighting health promotion and social mobilization. The Health Education Initiative will strengthen the health education system, popularize knowledge about health, teach scientifically valid perspectives of health, strengthen early intervention, promote healthy lifestyles in good ecological and social environments, and ultimately prolong healthy lives.

\section{KEY CONTENT \& TARGETS}

The Health Education Initiative has a premise that improvement of health literacy will improve the health of all people. That is, a prerequisite for improving health is to improve knowledge about health, which can help make healthy behaviors and skills high quality and practiced universally. Health education is the first of the 15 Healthy China Initiatives, and it works at three levels - the individual/family level, the societal level, and the governmental level - indicated in shorthand by " $1,2,7,7$ ". 1, 2, 7, 7".

"1" refers to an outcome indicator of health literacy that is measured at the national level.

"2" refers to two binding targets. The first is to establish a national and provincial database of health science experts and a national resource of health knowledge, with the purpose of establishing a mechanism of health science knowledge dissemination. The second target is to establish a health education and health promotion performance evaluation mechanism for medical institutions and medical personnel.

"7" and "7" refer to the contents of each of the seven parts of the individual/family and society/government levels.

Targets of the Health Education Initiative are to achieve national health literacy levels of at least $22 \%$ by 2022 and $30 \%$ by 2030 . Specific indicators are shown in Table 1.

Health literacy refers to the ability of individuals to access and understand basic health information and services, and use information and services to make informed decisions to maintain and promote their own health (4). The health literacy level refers to the proportion of people achieving health literacy among the total population.

Figure 1 shows health literacy over time. In 2008, health literacy was 6.48\% (5); between 2012 and 2019, health literacy increased. Literacy rose by $2.6 \%$ and $2.88 \%$ in 2016-2017 and 2017-2018, 2.11\% from 2018 to 2019.

We think that rising health literacy may be related to popularization and development of the Internet in 
TABLE 1. Major outcome indices proposed for the Health Education Initiative.

\begin{tabular}{|c|c|c|}
\hline \multirow{2}{*}{ Indices } & \multicolumn{2}{|c|}{ Targeted data } \\
\hline & $2020(\%)$ & $2030(\%)$ \\
\hline National level of health literacy & 22 & 30 \\
\hline Basic knowledge and conceptual literacy level & 30 & 45 \\
\hline Healthy lifestyle and behavioral literacy level & 18 & 25 \\
\hline Basic skills and literacy level & 20 & 30 \\
\hline Basic medical literacy & 20 & 28 \\
\hline Chronic disease prevention and control literacy & 20 & 30 \\
\hline Infectious disease prevention and control literacy & 20 & 25 \\
\hline Population blood donation rate & 15 & 25 \\
\hline Proportion of departments set up in traditional Chinese medicine hospitals to treat sub-health diseases & 90 & 100 \\
\hline
\end{tabular}

China. In 2016, fixed broadband networks and mobile communication networks - the foundation of China's communication industry - made great progress. The fully packetized fixed broadband network has been completed nationwide, and the $4 \mathrm{G}$ mobile communication network has been completed. China's network environment has been further optimized, as it encompasses the entire country and all cities with fiber optic networks. Currently, $72 \%$ of broadband is optical fiber, and there are over 700 million $4 \mathrm{G}$ users in China ( 6 ).

\section{STRATEGIES}

Achieving health literacy under the Healthy China Initiative will require implementation of several strategies, described below.

\section{Strengthen Monitoring and Testing}

Health literacy should be regularly monitored in a nationally-representative manner. In accordance with monitoring results, targeted science education can be conducted so that residents know, believe, and implement healthy behaviors, ultimately forming healthy values. The Healthy China Initiative advocates prevention first. Thus, it is important to disseminate science knowledge and inform residents about lifestyle changes that can prevent diseases at their sources. Once cultural values are established and firmly rooted, they will become behavioral habits and will improve the nation's overall health.

\section{Enrich Popular Science Knowledge Resources}

Strengthening science popularization and promoting

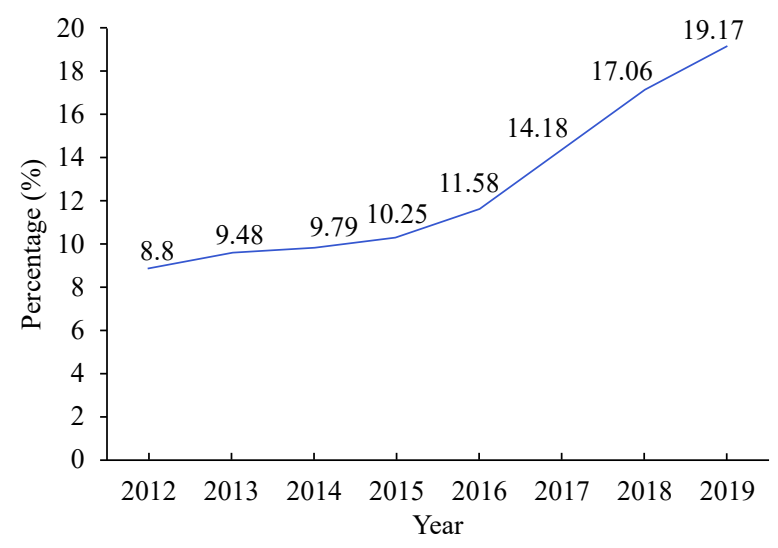

FIGURE 1. National health literacy level of residents in China, 2012-2019.

health knowledge requires analysis and effective resource material. For example, identifying behavioral change points and using plain language to explain professional concepts can make important knowledge accessible to lay people. To be effective, it is important to consider the audience when developing material to help people make decisions promoting healthy behaviors in their complicated lives. Health literacy materials must be updated to remain relevant and provide accurate information about hot or popular topics.

\section{Use Multiple Perspectives and Channels}

People receive information from a variety of sources. A key strategy is to combine new media to promote new ideas and knowledge from multiple perspectives and channels. At the government level, it is necessary to enhance inter-governmental cooperation and increase channels for health knowledge dissemination. For medical professionals, health industry and other 
associations should be encouraged to organize experts to for medical institution-oriented training. Communities should regard promotion of health knowledge as important work and organize health communication activities that address people's health problems. It is especially important to strengthen the promotion of health literacy among in poor areas.

\section{PERSPECTIVE}

Health literacy is an important factor for effective disease prevention and control. With COVID-19 under good control in China, health literacy will help the public maintain protection from the virus while fighting the pandemic globally. In view of resurgences of COVID-19 in other countries during autumn and winter this year, precautions must be taken in China. The COVID-19 outbreaks in Qingdao, Tianjin, and Shanghai remind us that we need to adhere to important prevention and control measures: wearing masks, maintaining safe physical distancing, washing hands frequently, and ventilating homes adequately. These measures are effective not only for COVID-19, but also for other respiratory infections such as influenza. Basic prevention and control measures need to be universally adopted so that everyone can master and apply them to better prevent and control COVID-19.
In the long run, it is important to increase healthy life expectancy to 79 years - a target that is on the pathway to achieving a health China by 2030 .

doi: $10.46234 / \mathrm{ccdcw} 2021.018$

\# Corresponding author: Wen Jiang, 2225840889@qq.com.

1 Media Integration and Mass Communication Department, China
Population Communication Center, Beijing, China.

Submitted: November 11, 2020; Accepted: January 01, 2021

\section{REFERENCES}

1. Central Committee of Chinese Communist Party, State Council. The plan for healthy China 2030. Beijing: Government of the People's Republic of China, 2016. http://www.gov.cn/zhengce/2016-10/25/ content_5124174.htm. [2020-12-12]. (In Chinese).

2. Department of Economic and Social Affairs, Unite Nations. Transforming our world: the 2030 agenda for sustainable development. https://sdgs.un.org/2030agenda. [2020-12-22].

3. Healthy China Action Promotion Committee. Healthy China initiative (2019-2030). http://www.nhc.gov.cn/guihuaxxs/s3585u/201907/e9275 fb95d5b4295be8308415d4cd1b2.shtml. [2020-12-22]. (In Chinese).

4. Chinese Center for Health Education. Health literacy monitoring report of Chinese residents. Beijing: People's Medical Publishing House. 2018. (In Chinese).

5. Wang P, Mao QA, Tao MX, Tian XY, Li YH, Qian L, et al. Survey on the status of health literacy of Chinese residents in 2008. Chin J Health Educ 2010;26(4):243 - 6. http://dx.doi.org/10.16168/j.cnki.issn.10029982.2010.04.021. (In Chinese).

6. Fu YH. Review of China's information and communication industries in 2016. Mobile Commun 2017;41(1):7 - 12. http://dx.doi.org/10.3969/ j.issn.1006-1010.2017.01.001. (In Chinese). 